Check for updates

Cite this: J. Anal. At. Spectrom., 2021, 36, 2056

Received 30th November 2020 Accepted 29th June 2021

DOI: 10.1039/d0ja00491j

rsc.li/jaas

\section{Quantitative manganese dissolution investigation in lithium-ion batteries by means of X-ray spectrometry techniques}

\author{
Claudia Zech, (D) *a Marco Evertz, ${ }^{\mathrm{b}}$ Markus Börner, ${ }^{\mathrm{b}}$ Yves Kayser, (D) a \\ Philipp Hönicke, (iD a Martin Winter, ${ }^{\text {bc }}$ Sascha Nowak iD ${ }^{b}$ and Burkhard Beckhoff ${ }^{a}$
}

Each battery suffers from degradation effects which lead to capacity fading and life cycle reduction. With electrochemical methods, the battery capacity decrease can easily be monitored, but they are inappropriate to comprehend the underlying chemical and physical properties responsible for the capacity fading. In the present paper, we demonstrate the power of X-ray spectrometry techniques to investigate the transition metal dissolution (TMD) fading process for manganese in Lithium lon Batteries (LIBs). Regarding aging processes, quantitative analyses are important to evaluate the magnitude of a certain process for the total capacity decrease. The challenge for the investigation of aged battery materials is the lack of reference materials such that techniques based on them cannot be applied. With the use of reference sample-free quantitative X-ray fluorescence analysis (XRF), we investigated the total mass deposition of manganese deposited at the anode for an aged cell. For 50 full cycles with elevated cut-off voltage, the capacity decreased by $12.5 \%$, while up to $1.6 \%$ of cathodic manganese was found deposited in the anode. Furthermore, the species of manganese deposited in the anode has been studied with near-edge X-ray absorption fine structure analysis (NEXAFS) at the manganese L-edges and K-edges. In addition to a different discrimination capability at L- and K-edges, the application of significantly different excitation energies provides sensitivity to different sample depths. We determined the manganese deposition to be in di- and tetra-valent states, whereby tetravalent manganese is solely found in the anode bulk. The combination of the different methods generates a clearer understanding of the chemical processes in the battery and can therefore help to improve the performance by exploring the absolute mass deposition and the electronic structure of elements contained in enhanced material combinations.

\section{Introduction}

One of the most promising compositions for cathodes for lithium ion batteries (LIBs) is layered lithium metal oxides, especially the ternary combination of nickel, cobalt and manganese $\mathrm{LiNi}_{1 / 3} \mathrm{Co}_{1 / 3} \mathrm{Mn}_{1 / 3} \mathrm{O}_{2}$ (NCM111) due to its tailored properties..$^{1-3}$ Despite all the advantages offered by LIBs, one major drawback is the decay of capacity with ongoing cell usage assigned to the degradation of battery cell components, especially the cathode material..$^{3-5}$ The solid electrolyte interphase (SEI) acting as a passivation film to inhibit further electrolyte reduction is of immense importance for the stability of LIB cells. ${ }^{4,6}$ For example in the case of $\mathrm{LiMn}_{2} \mathrm{O}_{4}$ (LMO) cathodes, it

${ }^{a}$ Physikalisch-Technische Bundesanstalt, Abbestr. 2-12, 10587 Berlin, Germany. E-mail: Claudia.zech@ptb.de

${ }^{b}$ University of Münster, Münster Electrochemical Energy Technology, Corrensstraße 46, 48149 Münster, Germany

'Helmholtz Institute Münster, IEK-12 FZ Jülich, Corrensstraße 46, 48149 Münster, Germany was reported that manganese dissolution deteriorates these beneficial properties on the carbonaceous anode side and, therefore, negatively affects the life cycle. It has been proposed that the dissolution of LMO - with manganese in the oxidation states +III and +IV in equal parts - follows two dissolution mechanisms which are either a disproportionation reaction: $2 \mathrm{Mn}^{3+} \rightarrow \mathrm{Mn}^{2+}+\mathrm{Mn}^{4+}$ or acidic corrosion, catalyzed by $\mathrm{HF}$ or other inorganic acids: $\mathrm{Mn}^{3+}+2 \mathrm{HF} \rightarrow \mathrm{MnF}_{2}+2 \mathrm{H}^{+} .^{7-10}$ These dissolution mechanisms were recently adapted to NCM layered cathode materials at elevated charge cut-off voltages $(>4.4 \mathrm{~V} v s$. $\left.\mathrm{Li} / \mathrm{Li}^{+}\right)$, despite their different electrochemical environments. In contrast to LMO, NCM cathodes contain the reactive trivalent manganese just in minor impurities, while they mainly consist of tetravalent manganese which has a stable oxidation state and is electrochemically inactive and therefore does not easily undergo disproportionation reactions or acidic dissolution induced by HF. ${ }^{11,12}$ Thus, the aging of NCM LIBs cannot be primarily caused by $\mathrm{Mn}^{3+}$ species.

For a deeper understanding of the transition metal dissolution (TMD) process in NCM LIBs it is highly important to 
investigate the oxidation state as well as the quantity of the formed manganese species deposited at the negative electrode.

We apply hereafter X-ray spectrometry techniques to investigate degradation effects after 50 cycles with a rate capability of $1 \mathrm{C}$ and a cut-off voltage of $4.6 \mathrm{~V}$ for a NCM111 cell in the delithiated state.

Former studies that investigated the aging mechanism in this system revealed different results:

Wandt et al. applied for the aged anode operando Mn K-edge NEXAFS (near-edge X-ray absorption fine structure analysis) with reference samples and found a manganese dissolution rate of roughly $0.0017 \%$ per cycle in NMC cells. ${ }^{13}$ Evertz et al. used total-reflection XRF (TXRF) and found after 100 cycles a manganese loss of $0.165 \%$ by investigating the separator and the anode. ${ }^{11}$ Therefore, the graphite anode had to be prepared with ball milling after cycling and an internal standard was used as a reference for quantification. Buchberger et al. applied prompt-gamma-activation-analysis (PGAA) and determined the loss of transition metal for the 3 metals together to be $0.77 \%$ after 325 cycles. $^{\mathbf{1 4}}$ We investigated the aged anode with reference-sample free, thus physically traceable X-ray fluorescence analysis. ${ }^{15}$

Regarding the oxidation state of deposited manganese in aged anodes, former analyses are somewhat ambiguous: ex situ Mn K-edge NEXAFS studies revealed that deposited manganese is in an elemental, ${ }^{16} \mathrm{di}-,{ }^{17}$ tri- or tetra-valent ${ }^{18}$ state. Wandt et al. found divalent manganese by applying operando Mn K-edge NEXAFS and suggested that elemental manganese is only formed during ex situ studies. ${ }^{13}$ We applied soft and tender Xrays to probe the manganese L-edge and K-edge absorption fine structure for revealing the manganese oxidation state in different depths of the aged anode.

\section{Experimental setup \& methods}

\subsection{Cell assembly and cycling procedure}

For the battery cell, a MesoCarbon MicroBead (MCMB) graphite/hard carbon composite (MTI Corporation) serves as the anode material and lithium nickel cobalt manganese oxide (NCM) (Toda Kogyo Corp.) as the cathode material. Both electrodes were prepared on a pilot plant at the University of Münster (MEET Battery Research Center). The organic solventbased electrode preparation process uses polyvinylidene difluoride (PVDF, KynarFlex® 761) as the binder dissolved in $N$ methyl-2-pyrrolidone (NMP) (99.5\%, Acros Organics) as the processing solvent for slurry preparation. The active mass loadings for the cathode and anode were set at $93 \%$ and $95 \%$ for NCM and MCMB, respectively. In addition, carbon black (99.7\%, SuperP®, Timcal) was added as a conductive agent. The slurries were coated on thin aluminum (cathode) and copper (anode) foil with an active mass dry thickness of $93 \mu \mathrm{m}$ and 79 $\mu \mathrm{m}$ for the cathode and anode, respectively.

For the constant current cycling investigation, a coin cell was assembled using electrode areas of $1.13 \mathrm{~cm}^{2}$ for the cathode and the anode, respectively. As a separator, hybrid non-woven fiber/ polypropylene foil (Freudenberg 2190/Celgard 2500) was used with an area of $2.67 \mathrm{~cm}^{2}$ and $100 \mu \mathrm{L}$ of $1 \mathrm{M} \mathrm{LiPF}_{6}$ in ethylene carbonate/ethyl methyl carbonate (EC/EMC, 50/50 wt\%) (LP 50, Selectilyte ${ }^{\circledR}$, BASF) solution acted as the electrolyte.

Cycling was performed using a Maccor series 4000 battery test system (Maccor Inc.) in a voltage window from 3.0-4.6 V. Furthermore, three SEI formation cycles at $32 \mathrm{~mA}(0.2 \mathrm{C})$ were carried out with subsequent 50 aging cycles using a specific current of $160 \mathrm{~mA}(1 \mathrm{C})$.

Afterwards, the aged cell in the discharged state was disassembled in an argon filled glovebox and the carbonaceous anode was washed with $1 \mathrm{ml}$ dimethyl carbonate (DMC) to rinse off electrolyte residues.

\subsection{Quantification of the elemental mass deposition with $\mathrm{X}$ - ray fluorescence analysis}

$\mathrm{XRF}$ is a conventionally used method for elemental analysis which has detection limits in the low ppm (parts per million) range and is therefore well suited to investigate the anode regarding transition metal depositions originating from the cathode, where mass depositions are typically in the sub- $\mu \mathrm{g}$ $\mathrm{cm}^{-2}$ region. The determination of the influence of the different fading processes on the overall capacity loss of the cell is accessible only by using quantitative methods. We applied therefor an approach with no need for reference samples for quantitative elemental speciation: reference- or referencesample-free XRF is based upon radiometric and dimensional knowledge of all instrumental and experimental parameters as well as good knowledge on X-ray fundamental parameters. Its physical traceability chain addresses the lack of (certified) reference materials or calibration specimens used as a reference sample in chemical traceability chains. ${ }^{15,19,20}$

The X-ray spectrometry measurements were performed in the PTB laboratory at the synchrotron radiation facility BESSY II in Berlin. All measurements were realized in an ultra-high vacuum (UHV) environment of around $2 \times 10^{-7}$ mbar. $^{20} \mathrm{We}$ used two different synchrotron radiation beamlines for the reported experiments. Soft X-ray experiments were carried out at the plane grating monochromator beamline ${ }^{21}$ and tender (hard) $\mathrm{X}$-ray experiments at the four crystal monochromator beamline. ${ }^{22}$ The measurement set-up used at both synchrotron radiation beamlines is shown in Fig. 1.

The mass deposition $\frac{m_{\mathrm{i}}}{F_{\mathrm{i}}}$ is calculated from the count rate of a fluorescence line of a given element $\mathrm{i}$ by using the transformed Sherman equation ${ }^{15}$

$$
\frac{m_{\mathrm{i}}}{F_{\mathrm{i}}}=\frac{-1}{\frac{\mu\left(E_{0}\right)}{\sin \vartheta_{0}}+\frac{\mu\left(E_{\mathrm{f}}\right)}{\sin \vartheta_{\text {out }}}} \ln \left\{1-\frac{N_{\mathrm{i}}\left(\frac{\mu\left(E_{0}\right)}{\sin \vartheta_{0}}+\frac{\mu\left(E_{\mathrm{f}}\right)}{\sin \vartheta_{\text {out }}}\right)}{\frac{N_{0}}{\sin \vartheta_{0}} \omega_{\mathrm{Xi}} g_{\mathrm{Xi}}\left(E_{\mathrm{f}}\right) \tau_{\mathrm{i}}\left(E_{0}\right) \frac{\Omega}{4 \pi}}\right\}
$$

where $\dot{N}_{0}$ represents the photon flux of the excitation photons with energy $E_{0}, \dot{N}_{\mathrm{i}}$ the photon flux of the detected fluorescence photons, $\Omega$ the solid angle of detection, $\vartheta_{0}$ the angle between the incident photon beam and the sample surface, $\vartheta_{\text {out }}$ the angle between the emitted fluorescence photons and the sample surface, $\omega_{\mathrm{Xi}}$ the fluorescence yield and $g_{\mathrm{Xi}}\left(E_{\mathrm{f}}\right)$ the transition probability for element $\mathrm{i}$ and subshell $\mathrm{X}$ for fluorescence 


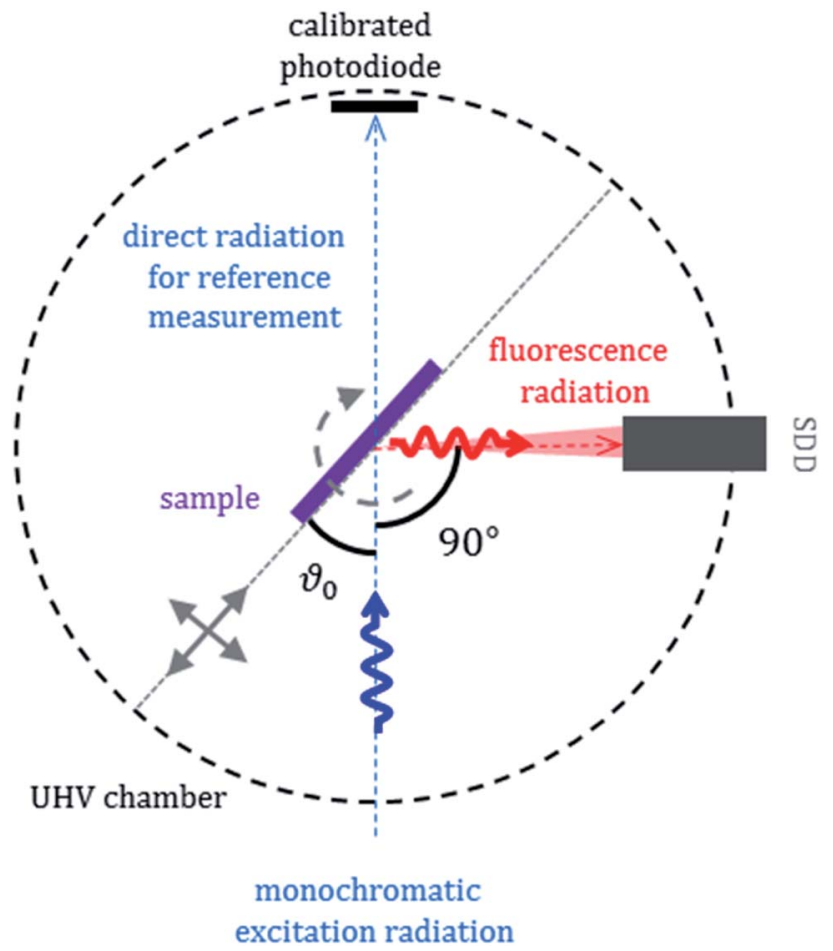

Fig. 1 Measurement setup for quantitative XRF and NEXAFS experiments.

energy $E_{\mathrm{f}} \cdot \mu$ is the total mass attenuation coefficient and $\tau_{\mathrm{i}}$ the partial photo ionization cross section. $\dot{N}_{0}$ is determined by measuring the direct beam current $I_{0}$ with a radiometrically calibrated photodiode with known energy-dependent efficiency $s_{\mathrm{D}}$.

$$
N_{0}=\frac{I_{0}}{s_{\mathrm{D}} E_{0}}
$$

The photon flux $\dot{N}_{\mathrm{i}}$ of fluorescence photons is calculated with the determined counts $N_{\mathrm{i}}$ from the spectrum deconvolution (see Fig. 1) using a known response function ${ }^{23}$ divided by integration time $t$ and energy-dependent detector efficiency $\varepsilon_{\mathrm{SDD}}$ of the calibrated silicon drift detector (SDD).

$$
N_{\mathrm{i}}=\frac{N_{\mathrm{i}}}{t \varepsilon_{\mathrm{SDD}}}
$$

For the fundamental parameters $\left(\mu, Q_{\mathrm{Xi}}, \tau_{\mathrm{i}}\right)$ either tabulated data are used or dedicated experiments are performed to determine the values in a physically traceable manner. ${ }^{\mathbf{2 4 , 2 5}}$ For the quantification of the manganese mass deposition, the fluorescence yield as well as photoionization cross sections were taken from the xraylib database. ${ }^{26}$

\subsection{Near-edge X-ray absorption spectrometry}

NEXAFS is a contactless and non-destructive technique and allows for element sensitive investigations of the unoccupied valence structure of atoms in the sample. From these investigations, the chemical species of an element can be derived. It is applicable to solid, liquid, gaseous, crystalline and amorphous structures, which is especially for battery materials an advantage since the structure of the active material often changes during the battery performance. NEXAFS measurements can be realized either in transmission or in fluorescence mode. The requirements for the incident radiation are highenergy resolution, energy-tunability and, in the case of measurements in fluorescence mode, a high incident flux. All parameters are typically fulfilled when using synchrotron radiation. For the presented measurements, the energy of the incident photons is varied in the energy range around the absorption edge and the resulting fluorescence photons are detected using an energy-dispersive detector. The intensity of the recorded X-ray fluorescence line is then proportional to the absorption cross section in the case of small sample thickness. The fine structure observed around the absorption edges is caused by the transitions of core electrons to unoccupied atomic or molecular orbitals. For the post-edge region scattering and interference of the electron potential with the Coulomb potential of neighboring atoms are the underlying processes modulating the measurement signal and provide coordination information. The near-edge resonances contain information about the valence states as well as atomic binding partner, binding distance and local structure. NEXAFS provides information about $1 \mathrm{~s} \rightarrow 4 \mathrm{p}$ transitions in the case of K-edge investigations and $2 \mathrm{p} \rightarrow 3 \mathrm{~d}$ transitions in the case of $\mathrm{L}_{3}, \mathrm{~L}_{2}$ edge investigations. Especially for transition metals such as manganese, L-edge NEXAFS is advantageous since it enables access to transitions to unoccupied electronic levels in the $3 \mathrm{~d}$ shell and determines the oxidation state of a species.

To investigate the oxidation state of the aged anode described above, materials with known manganese valence states - manganese(II)nitrate, manganese(III)oxide and manganese(Iv)oxide - were measured as reference NEXAFS compound samples for di-, tri- and tetra-valent manganese. While the references for tri- and tetravalent manganese (manganese(III)oxide and manganese(Iv)oxide) were measured as powders, droplets of manganese(II)nitrate in $2 \%$ nitride acid were applied on a Freudenberg separator. NEXAFS measurements for the aged anode, the pristine NCM cathode, and the reference NEXAFS compounds were performed at the $\mathrm{K}$ and the $\mathrm{L}_{3}, \mathrm{~L}_{2}$-edges of manganese. For the $\mathrm{L} 2$ and $\mathrm{L} 3$ edges, the emitted fluorescence was recorded while scanning the incident photon energy between $635 \mathrm{eV}$ and $660 \mathrm{eV}$ in $0.25 \mathrm{eV}$ energy steps. For the K-edge measurements, the incident photon energy range between $6525 \mathrm{eV}$ and $6600 \mathrm{eV}$ was monitored in $0.5 \mathrm{eV}$ steps for the near edge region and in $1 \mathrm{eV}$ steps for the post-edge region.

\section{Results and discussion}

The used cycling procedure for SEI formation and aging as well as the resulting capacity and efficiency are shown in Fig. 2.

After the cycling procedure, we determined a $12.5 \%$ capacity decrease.

The fluorescence spectrum of the aged anode irradiated with $\mathrm{X}$-ray photons with an energy of $8500 \mathrm{eV}$ and measured during 

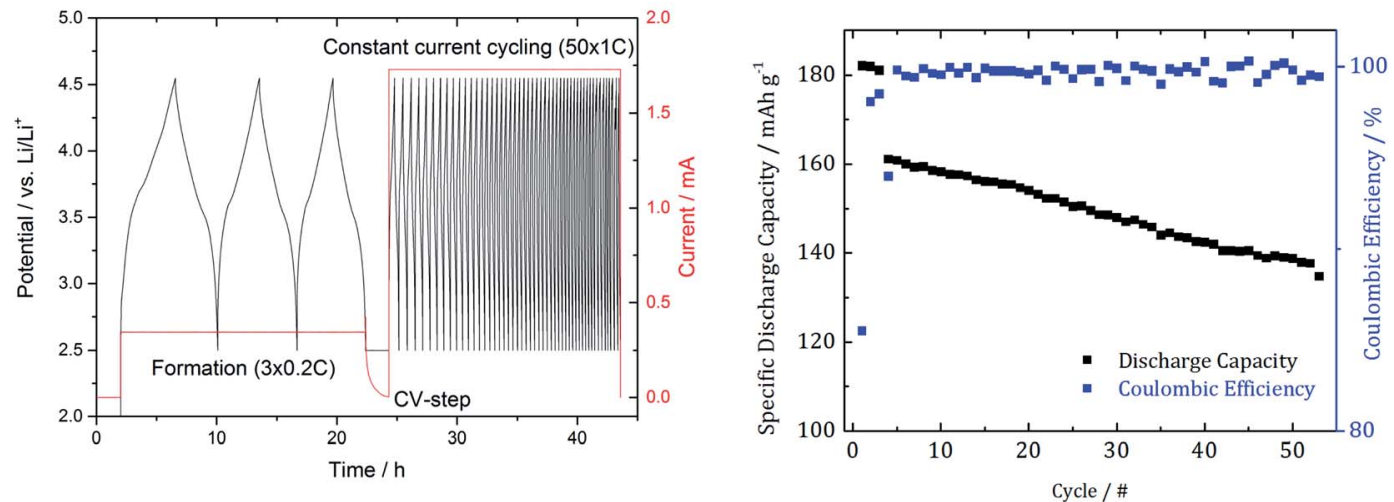

Fig. 2 Cycling procedure with $3 \mathrm{SEl}$ formation cycles and 50 subsequent cycles to induce degradation effects (left) as well as the resulting capacity and the coulombic efficiency (right).

a detector integration time of $800 \mathrm{~s}$ is shown in Fig. 3. Under these conditions, decent discrimination with the SDD between coherently scattered photons $E_{0}$ and $\mathrm{Mn} \mathrm{K} \alpha$ fluorescence photons is achieved. The various fluorescence lines originate from different elements in the cell components. While the phosphorus $\mathrm{K} \alpha$ line contribution originates from the conductive salt lithium hexafluorophosphate $\left(\mathrm{LiPF}_{6}\right)$ in the electrolyte, the fluorine $\mathrm{K} \alpha$ line originates from the polyvinylidene difluoride (PVDF) binder used in the electrode preparation as well as from the conductive salt $\mathrm{LiPF}_{6}$. The iron contributions occur since the incident photon energy is high enough to transmit through the whole anode and even excite the sample holder consisting of steel.

The detected count rate of the manganese K $\alpha$ fluorescence $N_{\mathrm{Mn}}$ is determined by means of spectral deconvolution which is essential to subtract the non-negligible contributions from Compton scattering and resonant Raman scattering of the excitation radiation. The deconvolution of XRF spectra based on experimentally determined detector response functions and using only physical contributions enables an advanced

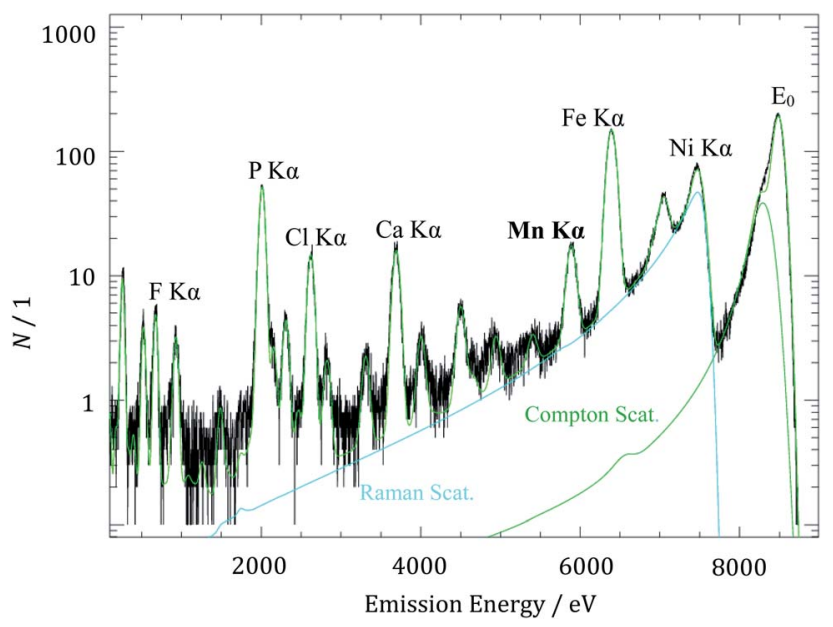

Fig. 3 X-ray fluorescence spectrum of the aged anode for the determination of manganese mass deposition with an excitation energy $E_{0}$ of $8500 \mathrm{eV}$. quantitation. The resonant Raman scattering of the incident photons at the copper K-edge is caused by the excited current collector foil which is located under the active carbon material of the anode. After solving the Sherman equation, a Mn mass deposition of $0.14 \mu \mathrm{g} \mathrm{cm}^{-2}$ was determined for the aged anode. With respect to the anode area and under the assumption of a homogeneous deposition, it corresponds to a total Mn mass of $0.16 \mu \mathrm{g}$ deposited on the entire anode.

The aim for the characterization of an aging effect is to correlate it with the capacity decrease. While the capacity decreased by $12.5 \%$ (Fig. 2), the amount of cathodic manganese deposited at the anode is in range of $0.03-0.16 \%$ assuming that the pristine NCM cathode contains typically about $100 \mu \mathrm{g}$ to 500 $\mu \mathrm{g}$ manganese per $\mathrm{cm}^{2}$. The findings of former quantitative studies by Wandt et al. ${ }^{13}$ Evertz et al. ${ }^{11}$ and Buchberger et al. ${ }^{14}$ mentioned in the Introduction part are within the range of our results.

Although only a low amount of $\mathrm{Mn}$ is transferred, an exact assessment of the impact on the observed capacity fading requires further investigation. While this insight only reflects the mass deposition after 50 cycles, and hence the occurrence of Mn dissolution and deposition, future ex situ or in situ timeresolved studies will allow for a more detailed insight into the exact mechanism.

Regarding the chemical speciation of $\mathrm{Mn}$, the NEXAFS results are shown in Fig. 4.

For the reference NEXAFS compounds, the absorption edge shifts to higher energies with a higher valence state, which is in accordance with theory. While the main peak for divalent manganese occurs around $6551 \mathrm{eV}$, for trivalent $\mathrm{Mn}$ it is at $6556 \mathrm{eV}$ and for tetravalent $\mathrm{Mn}$ at $6558 \mathrm{eV}$. For all samples a prepeak at $6540 \mathrm{eV}$ is detected, which can be attributed to the transition from $1 \mathrm{~s}$ to unoccupied $3 \mathrm{~d}$ orbitals or valence shells. Especially for compounds of manganese with oxygen, there is a significant pre-peak because of $\mathrm{Mn} 3 \mathrm{~d}-\mathrm{O} 2 \mathrm{p}$ orbital mixing. ${ }^{27}$ For tetravalent manganese, like in the NCM cathode, a pre-peak is found at $6543 \mathrm{eV}$; hereby, the intensity of the pre-peak provides insights into the molecule coordination.

$\mathrm{L}_{3}$-edge resonances between $639 \mathrm{eV}$ and $645 \mathrm{eV}$ result from $2 \mathrm{p}_{3 / 2}$ to $3 \mathrm{~d}$ transitions and $\mathrm{L}_{2}$-edge resonances between $652 \mathrm{eV}$ 

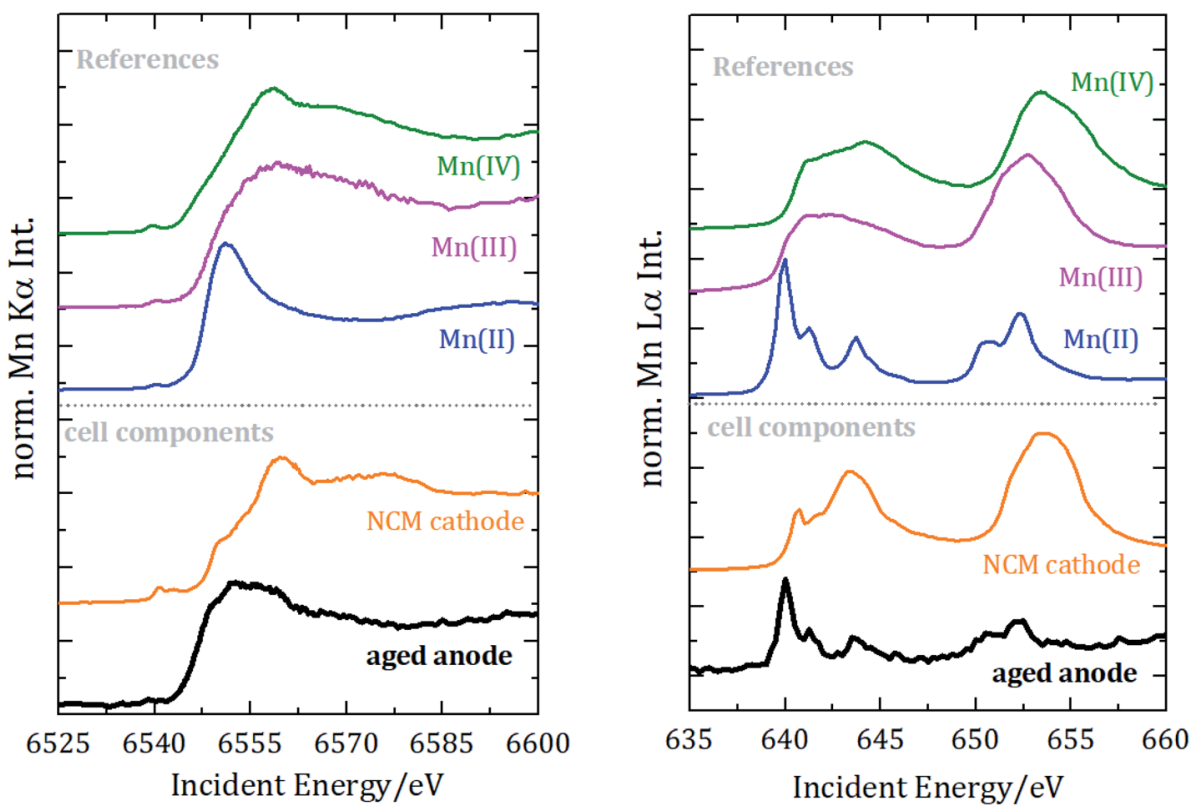

Fig. 4 Near-edge $X$-ray absorption spectrometry of manganese for the $K$-edge (left) and the $L_{2}$ und $L_{3}$ edges (right). Spectra are vertically shifted for better visualization.

and $656 \mathrm{eV}$ result from $2 \mathrm{p}_{1 / 2}$ to $3 \mathrm{~d}$ transitions. The resonance at $640 \mathrm{eV}$ is often used as an indicator for divalent manganese. For higher oxidation states, the L-edge resonances shift to higher energies.

To identify the distribution of the oxidation state of the aged anode, linear combination of the three reference spectra has been performed for both NEXAFS measurements, see Fig. 5 .

The best fit for the K-edge NEXAFS spectra of the aged anode is composed of $59 \%$ divalent and $41 \%$ tetravalent manganese. In contrast, the spectrum for the L-edges is best approximated by divalent manganese solely. The different peak ratios in the Ledge spectra of the anode and the divalent reference could be explained by the different sample environments and thus various self-absorption contributions as manganese(II)nitrate is aqueous and dropped on separator foil. The incorporation of divalent manganese into the SEI results in either blocking of lithium ion diffusion paths into/from carbonaceous materials and/or growth of an anode SEI by further decomposition of the electrolyte, both resulting in capacity decay.

Information derived from L-edge and K-edge NEXAFS measurements originates from different depths in the anode because of the significantly different exciting photon energies. ${ }^{28}$ While the entire anode thickness can be analyzed with K-edge NEXAFS, the maximum penetration depth in the case of Ledge investigation is around a few hundred $\mathrm{nm}$. Thus, we can conclude that tetravalent manganese is only formed inside the anode, while in the region near the surface only divalent manganese is present. The combination of Mn L-edge and $\mathrm{Mn}$ K-edge NEXAFS therefore allows for discriminating the chemical state of $\mathrm{Mn}$ in different sample volumes or probing depths.
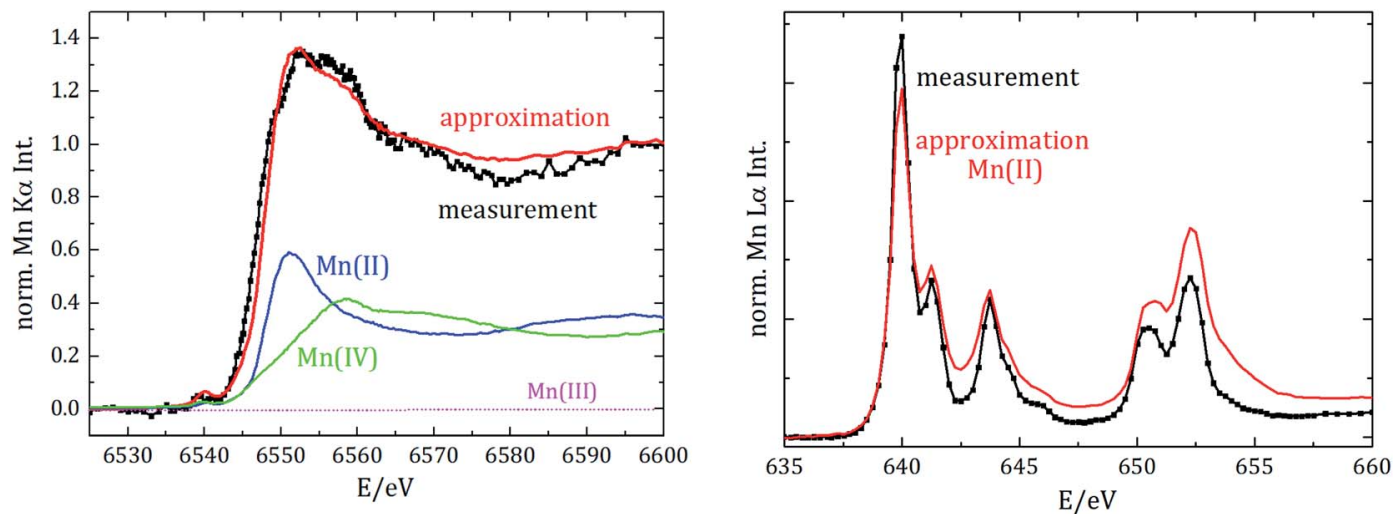

Fig. 5 Results of the linear combination of reference spectra to approximate the spectrum of the aged anode (left: for the K-edge NEXAFS analysis; right: for the $L_{2}, L_{3}$ edge NEXAFS). The measurement of the aged anode is shown in black, and the best approximation in red. While the spectrum of L-edge NEXAFS is best approximated by divalent manganese solely, the K-edge approximation is composed of di- and tetra-valent components (blue and green graphs). 
Moreover, we did not detect any trivalent manganese in the anode. Since the anode was investigated after the battery was completely discharged, we additionally analyzed whether divalent manganese could be further reduced due to the negative potential. Therefore, manganese foil was measured as the reference NEXAFS material for elemental manganese and spectral deconvolution with linear combination approximation was repeated, but we did not detect any elemental manganese in the anode.

\section{Conclusion}

We applied reference sample-free X-ray spectrometry as a traceable method to quantify lost manganese as ageing products at the anode surface. We found that after 50 cycles up to $1.6 \%$ of the cathodic manganese has been deposited at the anode, while the capacity decreased by $12.5 \%$ in agreement with former quantitative analyses.

With high-resolution X-ray absorption spectrometry, we could identify the oxidation states of the degradation products in LIBs, which is crucial to know since various species undergo different reactions. For the information of the species of manganese, we measured the X-ray absorption fine structure for a set of different manganese containing components with oxidation states between +II and +IV. Mn deposited at the aged MCMB anode was found to be di- and tetra-valent. With the combination of soft (Mn-L edges) and tender X-ray radiation (Mn K-edge), we could reveal that tetravalent manganese is solely present in the anode bulk, while divalent manganese is found both in the bulk volume and close to the anode surface. So, as for LMO cells, divalent Mn species are found in the anode of NCM cells although the cathode does not contain reactive trivalent Mn. In addition, we did not detect any elemental manganese or trivalent manganese in the aged anode.

Further quantitative investigations, especially for the electrolyte, would be helpful to understand the transport mechanism of the transition metals from the positive to the negative electrode. The next step will be to perform these measurements under in situ or even operando conditions to avoid perturbations or modifications by cell opening. While the amount of manganese in the anode should not differ from the used analysis mode, the results for the oxidation state from ex situ investigations should be further validated by operando characterization since results from different groups differ significantly.

Finally, we would like to emphasize that our methodological approach can be readily applied for the investigation of further transition metals like nickel and cobalt relying on the good knowledge of their fundamental parameters.

\section{Conflicts of interest}

No conflict to declare.

\section{Acknowledgements}

This research was funded by the German Science Foundation (DFG Project ISIBAT Sta 324/54). This research was performed within the EMPIR project ADVENT. This project has received funding from the EMPIR programme co-financed by the Participating States and from the European Union's Horizon 2020 Research and Innovation Programme.

\section{References}

1 M. Hu, X. Pang and Z. Zhou, J. Power Sources, 2013, 237, 229.

2 P. He, H. Yu, D. Li and H. Zhou, J. Mater. Chem., 2012, 22(9), 3680.

3 J. Kasnatscheew, M. Evertz, B. Streipert, R. Wagner, R. Klöpsch, B. Vortmann, H. Hahn, S. Nowak, M. Amereller, A.-C. Gentschev, P. Lamp and M. Winter, Phys. Chem. Chem. Phys., 2016, 18(5), 3956.

4 J. Vetter, P. Novák, M. R. Wagner, C. Veit, K.-C. Möller, J. O. Besenhard, M. Winter, M. Wohlfahrt-Mehrens, C. Vogler and A. Hammouche, J. Power Sources, 2005, 147(1-2), 269.

5 M. Broussely, P. Biensan, F. Bonhomme, P. Blanchard, S. Herreyre, K. Nechev and R. J. Staniewicz, J. Power Sources, 2005, 146(1-2), 90.

6 M. Gauthier, T. J. Carney, A. Grimaud, L. Giordano, N. Pour, H.-H. Chang, D. P. Fenning, S. F. Lux, O. Paschos, C. Bauer, F. Maglia, S. Lupart, P. Lamp and Y. Shao-Horn, J. Phys. Chem. Lett., 2015, 6(22), 4653.

7 C. Zhan, J. Lu, A. Jeremy Kropf, T. Wu, A. N. Jansen, Y.-K. Sun, X. Qiu and K. Amine, Nat. Commun., 2013, 4, 2437.

8 H. Zheng, Q. Sun, G. Liu, X. Song and V. S. Battaglia, J. Power Sources, 2012, 207, 134.

9 M. Wohlfahrt-Mehrens, C. Vogler and J. Garche, J. Power Sources, 2004, 127(1-2), 58.

10 L. Hanf, J. Henschel, M. Diehl, M. Winter and S. Nowak, Electrophoresis, 2020, 41(9), 697.

11 M. Evertz, F. Horsthemke, J. Kasnatscheew, M. Börner, M. Winter and S. Nowak, J. Power Sources, 2016, 329, 364.

12 M. Evertz, C. Lürenbaum, B. Vortmann, M. Winter and S. Nowak, Spectrochim. Acta, Part B, 2015, 112, 34.

13 J. Wandt, A. Freiberg, R. Thomas, Y. Gorlin, A. Siebel, R. Jung, H. A. Gasteiger and M. Tromp, J. Mater. Chem. A, 2016, 4(47), 18300.

14 I. Buchberger, S. Seidlmayer, A. Pokharel, M. Piana, J. Hattendorff, P. Kudejova, R. Gilles and H. A. Gasteiger, J. Electrochem. Soc., 2015, 162(14), A2737-A2746.

15 B. Beckhoff, J. Anal. At. Spectrom., 2008, 23(6), 845.

16 S. R. Gowda, K. G. Gallagher, J. R. Croy, M. Bettge, M. M. Thackeray and M. Balasubramanian, Phys. Chem. Chem. Phys., 2014, 16(15), 6898.

17 C. Delacourt, A. Kwong, X. Liu, R. Qiao, W. L. Yang, P. Lu, S. J. Harris and V. Srinivasan, J. Electrochem. Soc., 2013, 160(8), A1099-A1107.

18 R. Jung, F. Linsenmann, R. Thomas, J. Wandt, S. Solchenbach, F. Maglia, C. Stinner, M. Tromp and H. A. Gasteiger, J. Electrochem. Soc., 2019, 166(2), A378-A389.

19 B. Beckhoff, A. Gottwald, R. Klein, M. Krumrey, R. Müller, M. Richter, F. Scholze, R. Thornagel and G. Ulm, Phys. Status Solidi B, 2009, 246(7), 1415. 
20 J. Lubeck, B. Beckhoff, R. Fliegauf, I. Holfelder, P. Hönicke, M. Müller, B. Pollakowski, F. Reinhardt and J. Weser, Rev. Sci. Instrum., 2013, 84(4), 45106.

21 F. Senf, U. Flechsig, F. Eggenstein, W. Gudat, R. Klein, H. Rabus and G. Ulm, J. Synchrotron Radiat., 1998, 5(Pt 3), 780.

22 M. Krumrey, J. Synchrotron Radiat., 1998, 5(1), 6.

23 F. Scholze and M. Procop, X-Ray Spectrom., 2009, 38(4), 312.

24 R. Unterumsberger, P. Hönicke, J. L. Colaux, C. Jeynes, M. Wansleben, M. Müller and B. Beckhoff, J. Anal. At. Spectrom., 2018, 33(6), 1003.
25 R. Unterumsberger, P. Hönicke, B. Pollakowski-Herrmann, M. Müller and B. Beckhoff, Spectrochim. Acta, Part B, 2018, 145, 71.

26 T. Schoonjans, A. Brunetti, B. Golosio, M. Sanchez del Rio, V. A. Solé, C. Ferrero and L. Vincze, Spectrochim. Acta, Part $B$, 2011, 66(11-12), 776.

27 P. Thakur, K. H. Chae, M. Subramanain, R. Jayavel and K. Asokan, J. Korean Phys. Soc., 2008, 53(9(5)), 2821.

28 F. Reinhardt, B. Beckhoff, H. Eba, B. Kanngiesser, M. Kolbe, M. Mizusawa, M. Müller, B. Pollakowski, K. Sakurai and G. Ulm, Anal. Chem., 2009, 81(5), 1770. 